\title{
Gapping: A New LFG Approach
}

\author{
Yasir Hameed Alotaibi ${ }^{1}$ \\ ${ }^{1}$ Prince Sattam bin Abdulaziz University, Al Kharj, Saudi Arabia \\ Correspondence: Yasir Hameed Alotaibi, Prince Sattam bin Abdulaziz University, Al Kharj, Saudi Arabia. E-mail: \\ yasirho@hotmail.co.uk
}

Received: July 10, 2017 Accepted: July 28, 2017 Online Published: September 25, 2017

doi:10.5539/ijel.v7n6p188

URL: http://doi.org/10.5539/ijel.v7n6p188

\begin{abstract}
This paper discusses gapping in Modern Standard Arabic (MSA) and analyzes this phenomenon through the lexical-functional grammar (LFG) framework. It argues against previous analyses of nonconstituent coordination in LFG, including those proposed by Maxwell \& Manning (1996) and Frank (2002) and her followers. The main problem with those analyses is that they violate one of the well-formedness conditions in LFG, which requires the functional structure (f-structure) to be coherent. Therefore, the paper provides a new analysis of gapping in LFG, in which the deleted verb in the second conjunct is analyzed as a null verb that has a PRED value and that indicates the tense in its local clause.
\end{abstract}

Keywords: gapping, lexical functional grammar, nonconstituent coordination

\section{Introduction}

Gapping, an important syntactic phenomenon that has attracted attention in the literature, has been explored in a variety of syntactic theories and analyses. A simple example of gapping occurs when a coordinate structure that has two or more conjuncts; only the first conjunct has all its elements, including the predicate; and the subsequent conjuncts lack the predicate, which is understood from the predicate in the first conjunct, with or without other elements. Some examples of gapping are provided in the next section. The current paper focuses on an analysis of gapping in LFG, which is a non-transformational theory. We review previous analyses of gapping in LFG and argue against them in this paper, providing a new analysis to account for gapping and to avoid the problems encountered in previous analyses.

Maxwell \& Manning (1996) conduct the main analysis that attempts to account for non-constituent coordination, including gapping, in LFG. In this analysis, they assume that a phrase such as a VP or IP can be divided into two parts: the first part contains the verb in the first conjunct, and the second part contains the two complements in the coordinate structure. Thus, the verb in the first conjunct can be zipped to the complement in the first conjunct and then in the second conjunct. This analysis will be discussed and criticized in this paper.

The second analysis of LFG that attempts to account for non-constituent coordination is so-called function spreading, which is adopted by Frank (2002) and Sadler (2006), among others. In this analysis, some features of the first conjunct in a coordinate structure are assumed to be potentially spread over the entire coordinate structure. In the case of gapping, the PRED value of the verb and the tense should be assumed to be spread from the first conjunct over the entire construction. This analysis will be discussed in a later section.

This paper is organized as follows: Section 2 explains gapping and provides examples of this phenomenon in MSA. Section 3 discusses previous analyses of gapping in LFG, providing an introduction to analyze coordinate structures in LFG and then reviewing the analyses of non-constituent coordination in LFG, which are split analysis and function spreading analysis; both analyses are shown to have difficulty in accounting for gapping. Section 4 provides a new analysis of gapping: the deleted verb in the second conjunct is analyzed as a null verb that should have PRED and tense values in the second conjunct. This section provides plausible reasons for supporting the new analysis in this paper.

\section{Gapping}

Gapping is a syntactic phenomenon that has been discussed in the literature by researchers such as Gleitman (1965) and Lakoff \& Ross (1970). In this phenomenon, the first conjunct in a coordinate structure contains all its elements, while the subsequent conjuncts lack a predicate with or without other elements, which can be 
understood from the first conjunct. The following English example illustrates gapping, where the verb ate appears in the first conjunct but does not appear in the second conjunct because the verb is understood from the first conjunct.

(1) John ate fish and David chicken.

Gapping is also possible in MSA and other Arabic dialects. In MSA, a verb or copula can be deleted from non-initial conjuncts in a coordinate structure, as shown below:
a. qābala
fāris-un hāāmid-an
wa zayd-un
Payma-an.
Meet.PFV.3SGM Faris-NOM Hamed-ACC and Zayd-NOM Ayman-ACC

"Faris met Hamed and Zayd Ayman"
b. kāna
zayd-un
qā?im-an
wa
ḥāmid-un
ğālis-an.
be.PFV.3SGM
Zayd-NOM
standing.A-PTCP.3SGM-ACC and
Hamed-NOM
seated.A-PTCP.3SGM-ACC

"Zayd was standing and Hamed seated"

The two examples above illustrate gapping in a coordinate structure in MSA. Both contain two conjuncts, and the predicate is missing in the second conjunct in both constructions. In (2.a), the verb qäbala "met" appears in the initial conjunct and requires two arguments, a subject and an object, which are available in this conjunct. The subject carries nominative case marking, and the object carries accusative case marking, which are obligatory cases for the subject and the object in MSA. The second conjunct lacks an overt predicate, even though two arguments appear in this clause, which have the same case markings carried by the arguments in the first conjunct. Example (2.b) is similar to the previous example in that the second conjunct lacks an overt predicate, but it is different in terms of the kind of predicate that appears in the first conjunct. This predicate is a copula, namely, kāna 'to be'. Kāna in MSA is a predicate that requires two arguments: a subject and a complement. The subject carries nominative case marking, and the complement carries accusative case marking. The two arguments that appear in both conjuncts carry both cases. Notably, in both examples above, the deleted verbs in the second conjuncts can appear, and the examples would then be grammatical, as shown below:
(3) a. qābala
făris-un
hāmid-an
wa qābala
zayd-un Payma-an.
Meet.PFV.3SGM Faris-NOM Hamed-ACC an
Meet.PFV.3SGM
Zayd-NOM
Ayman-AC

"Faris met Hamed and Zayd met Ayman"
b. kāna
zayd-un
qā?im-an
wa kāna be
ḥāmid-un ğālis-an.
PFV.3SGM
Zayd-NOM
standing.A-PTCP.3SGM-ACC
and be.PFV.3SGM
Hamed-NOM

"Zayd was standing and Hamed was seated"

\section{Previous Analyses in LFG}

Before we review previous analyses of gapping in LFG, we should start with a brief overview of analyzing coordination in LFG. LFG has two levels of presentation: the constituent structure (c-structure) and the functional structure (f-structure). LFG is similar to other theories in assuming that words are organized into constituents, which are represented in a tree that is licensed by rules. The f-structure displays function information in LFG. The f-structure contains a set of pairs, where the first member of the pair is an attribute and the second member is its value (see Kaplan \& Bresnan, 1982; Dalrymple, 2001; Bresnan, 2001).

Bresnan et al. (1985) and Kaplan \& Maxwell (1988) examined the analysis of coordinate structures in LFG. This paper shows the analysis of sentential coordination in LFG, which is related to discussions of gapping. The simple coordinate structure between two sentences is shown in example (4). In this case, the coordination is assumed to be between two IPs, as shown in (5). The annotation beneath both IPs $\downarrow \epsilon \uparrow$ indicates that they are members of a set in the f-structure. This set corresponds to the mother IP, which is represented on the right-hand side in (5). The annotation $\uparrow=\downarrow$ beneath the conjunction (conj) means that this node is the same as the mother node. The rule in (5) licenses the tree in (6.a) below. The f-structure in (6.b) represents the function information, and both conjuncts are represented as members of the set corresponding to the mother IP. The conjunction in this f-structure is not part of the set; thus, it is a distributive feature that is distributed over all members of the set. The embedded f-structure $j$ represents the first conjunct in the coordinate structure, and the embedded f-structure $y$ represents the second conjunct.

(4) David studied and Richard slept. 
(5) IP

$\longrightarrow \begin{array}{ccc}\mathrm{IP}+ & \text { conj } & \mathrm{IP} \\ \downarrow \in \uparrow & \uparrow=\downarrow & \downarrow \epsilon \uparrow\end{array}$

(6) a.

IP

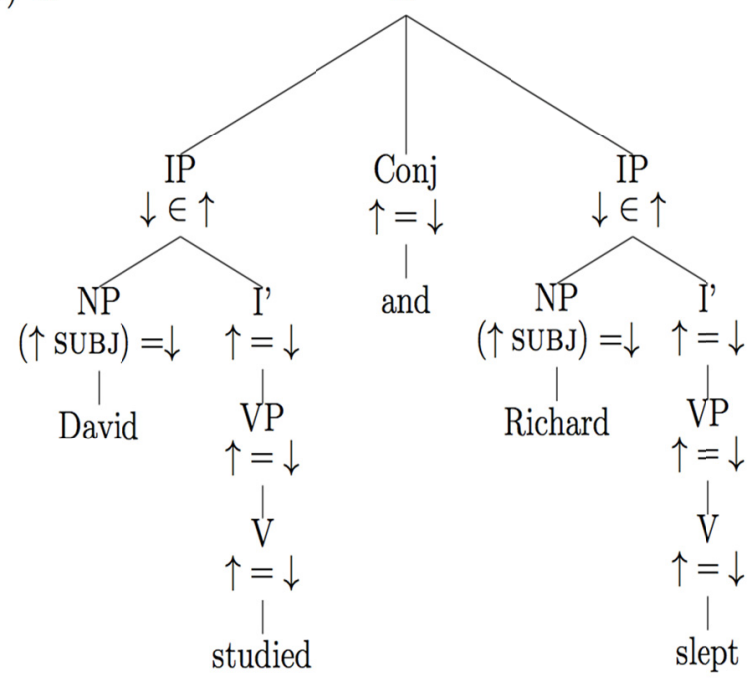

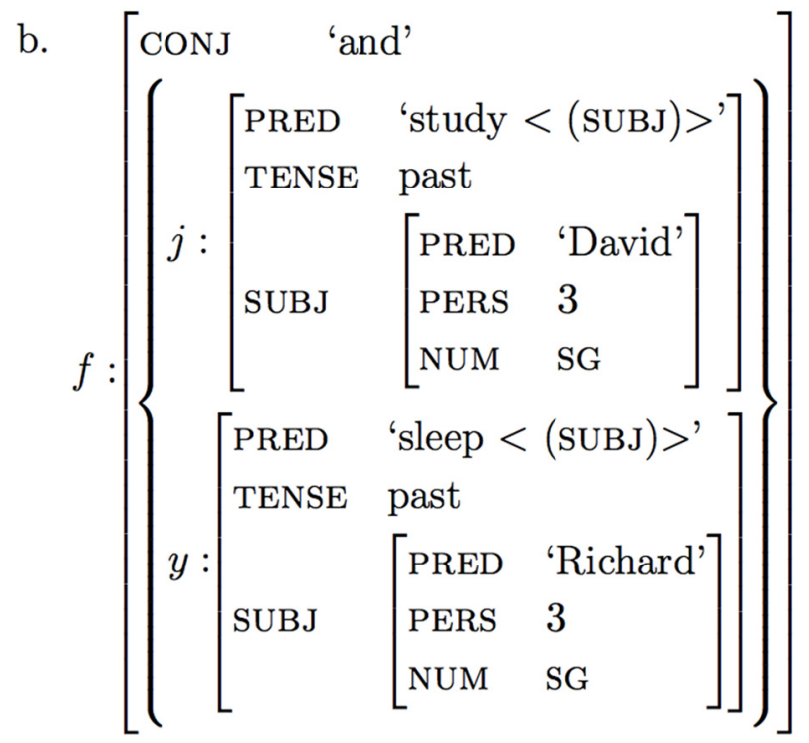

In LFG, some analyses are proposed to account for non-constituent coordination, and this phenomenon should include gapping. In this paper, the first analysis - proposed by Maxwell and Manning (1996) - is referred to as split analysis. The second analysis - used by Frank (2002) and Sadler (2006) - is called function spreading. This paper will review both analyses below.

\subsection{Split Analysis}

Maxwell \& Manning (1996) attempt to account for non-constituent coordination in general. An example such as (7), which illustrates gapping in English, should be accounted for in their analysis. Maxwell and Manning (1996) propose that this type of example is grammatical because the elements in the second conjunct constitute a valid completion of a VP that begins with the verb in the first conjunct. Hence, in example (7), the verb introduced in the first conjunct requires an object and an oblique, both of which are available in the first conjunct; however, the second conjunct contains another valid completion. Therefore, Maxwell \& Manning (1996) allow the splitting of the VP headed by the verb (also see Dalrymple, 2001).

(7) John introduced David to Mary and Louisa to Chris. 
The following phrase structure rules are suggested to show the VP division. In this analysis, the rule refers to a part of the phrase structure constituent. According to the following rules, the VP is broken into two parts: VP-x and $\mathrm{x}-\mathrm{VP}$.

(8) VP

$$
\longrightarrow \mathrm{VP}-\mathrm{x} \quad \mathrm{x}-\mathrm{VP}
$$

In analyzing example (7) above, one should assume that the first part of the VP analyzes the V and that the second part analyzes the complement, which contains the NP and PP, as shown in (9) below:

(9) a. VP-x $\longrightarrow V$

b. $\mathrm{x}-\mathrm{VP} \longrightarrow \mathrm{NP} \quad \mathrm{PP}$

In this analysis, the expressions on the right-hand side of the phrase structure rules can be shown as finite-state automaton, where the automaton can stop at a specific state in a phrase structure rule and then continue to analyze each conjunct.

The previous rules in (8) and (9) license the following c-structure to analyze example (7):

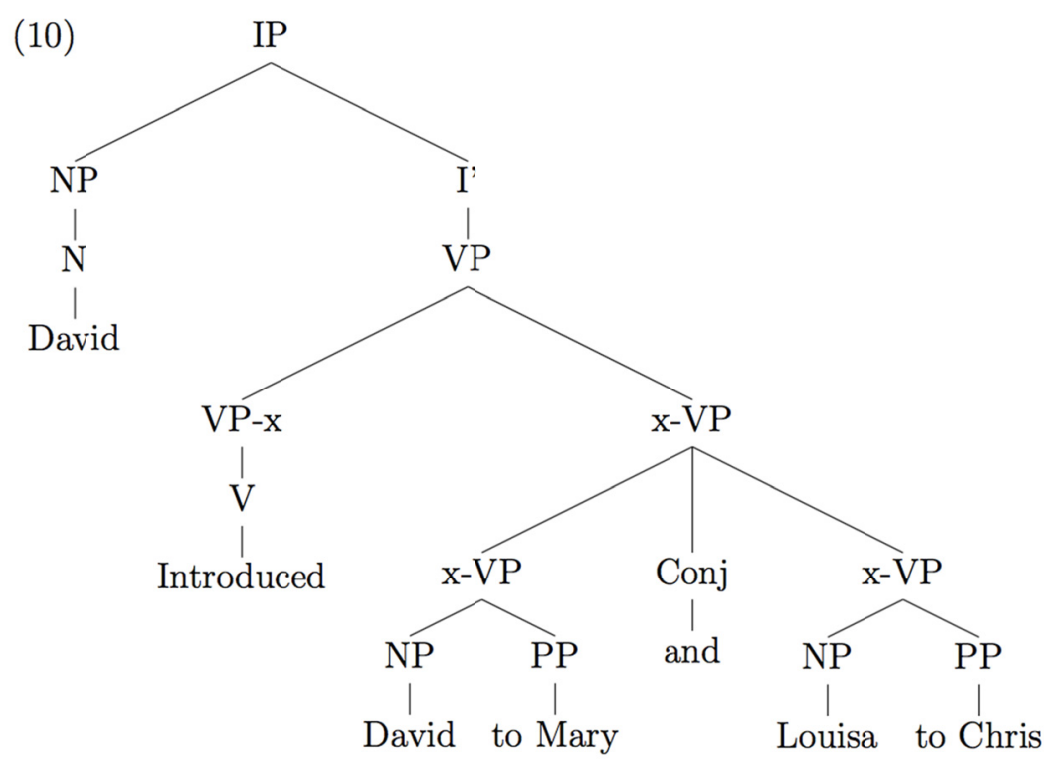

In this analysis shown in the tree above, the verb in VP-x can be zipped to the complement in the first conjunct $(\mathrm{x}-\mathrm{VP})$ or to the complement in the second conjunct $(\mathrm{x}-\mathrm{VP})$, and both are valid complements of this verb. In this analysis, VP-x stops at a particular point, which is where $\mathrm{x}-\mathrm{VP}$ starts.

Notably, this analysis assumes that the subject of the first conjunct in example (7) is shared between the two conjuncts and that the verb is also shared between them. The f-structure below shows the function information of this example, as suggested in this analysis. 


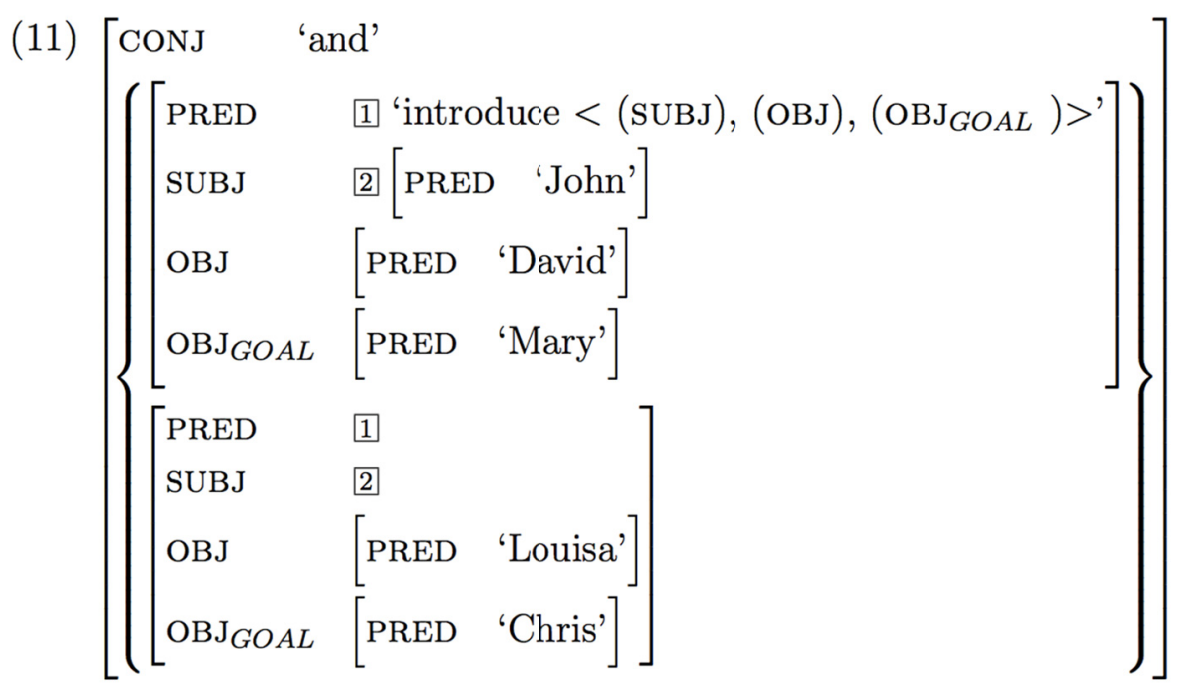

The f-structure above shows that this example contains two coordinated sentences, but the two sentences share one subject and one verb, which appear in the first conjunct. Number 1 in both embedded f-structures indicates that the two embedded f-structures share the same predicate, which is the verb introduce. This verb requires a subject and two objects, and these requirements are satisfied in both f-structures.

However, this analysis is problematic in predicting that one predicate can be shared by two f-structures, requiring the same arguments in each f-structure. In our view, this analysis finds that the verb introduce, which requires three arguments, has six arguments. A different analysis of gapping that can solve this problem will be proposed below.

Additionally, the analysis of Maxwell \& Manning (1996) may account for an example of gapping such as that presented in (7), but it cannot account for all examples of this phenomenon. For instance, the Maxwell and Manning (1996) approach cannot be used to analyze the example below. This example illustrates gapping in English and in other languages, including MSA, and the problem with this example is that the second conjunct has a different subject. Therefore, we need to break the IP, and then the verb, which is assumed to be shared between the two conjuncts, cannot be located between the subject and object in the second conjunct.

(12) John likes Mary and David Sue.

\subsection{Function Spreading}

Frank (2002) proposed function spreading analysis in LFG to analyze a type of nonconstituent coordination in German. This construction contains only one subject in the coordinate structure, which occurs in the leftmost conjunct. The second conjunct has no overt subject, but the subject in the first conjunct serves as the subject of this conjunct. Frank (2002) calls this phenomenon Subject Gap in Finite/Fronted (SGF). She provides the following example of SGF (2002, p. 176):

(13) "In den Wald ging der Jäger und ng einen Hasen".

into the forest went the hunter and caught a hare

"The hunter went into the forest and caught a hare"

The second conjunct in example (13) contains a verb, which requires a subject, but the subject is missing in this conjunct. Frank (2002) solves this problem through function spreading analysis, where the subject of the first conjunct can be spread over the entire construction. Frank (2002) proposes the following rule (Note 1) in (14), and she argues that this rule solves the problem of example (13), allowing the subject of the first conjunct, the hunter, to be distributed over the entire coordinate structure; in this way, the hunter fills the subject function in the second conjunct. In this rule, a coordinate structure between CPs is assumed. The GDF beneath the first CP refers to grammaticalized discourse functions: TOPIC, FOCUS and SUBJ. Thus, the annotation ( $\downarrow$ GDF $)=(\uparrow$ GDF) implies that a GDF function from the first CP is distributed over the entire construction, and it is the subject in example (13). 
(14) $\mathrm{CP}$

$\begin{array}{rlr}\longrightarrow \mathrm{CP} & \text { Conj } & \text { CP } \\ \downarrow \in \uparrow & \uparrow=\downarrow & \downarrow \epsilon \uparrow \\ (\downarrow \mathrm{GDF})=(\uparrow \mathrm{GDF}) & & \end{array}$

Additionally, Sadler (2006) uses the function spreading approach to account for some Welsh examples of coordination, where a coordinate structure contains one verb in one conjunct marked for tense and the other verbs are non-finite. Additionally, the subject of this construction, which appears in the tensed conjunct, is shared by all conjuncts. Sadler (2006) uses the following example to show this construction in Welsh.
(15) "Aeth
$\mathrm{y}$
ffermwr
at y drws
a churo
arno".
go-PAST.3SG
the farmer
to the door
and knock
on-3SM

"The farmer went to the door and knocked on it" (Rouveret, 1994, p. 302)

In example (15), the finite verb aeth ('went') appears in the first conjunct, and the past tense denoted by this verb is used in the second conjunct, which contains the non-finite verb churo ('knock'). In her analysis, Sadler (2006) aims to distribute the tense and the subject from the first conjunct to the other conjuncts in the coordinate structure. Sadler $(2006,1795)$ uses function spreading analysis to achieve this goal and proposes the following phrase structure rule:

(16) IP

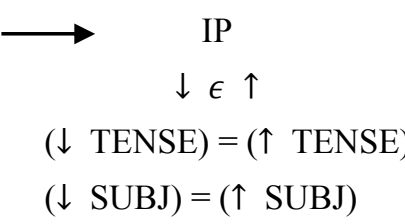

$$
\begin{array}{ll}
\text { Conj } & \text { VP } \\
\uparrow=\downarrow & \downarrow \in \uparrow
\end{array}
$$

In (16), coordination between an IP and a VP is assumed in this construction. The annotation beneath the first IP, which is $(\downarrow$ TENSE $)=(\uparrow$ TENSE $)$ and $(\downarrow$ SUBJ $)=(\uparrow$ SUBJ $)$, spread the tense and the subject of the first conjunct over the entire coordinate structure.

Function spreading analysis can be used to analyze gapping; thus, the verb in the first conjunct should spread over the entire coordinate structure. Alzaidi (2010) argues that this approach is the best way of analyzing gapping in the Hijazi dialect. Alzaidi $(2010,81)$ proposes the phrase structure rule in (18) for the example in (17), which is similar to the rules proposed by Frank (2002) and Sadler (2006).

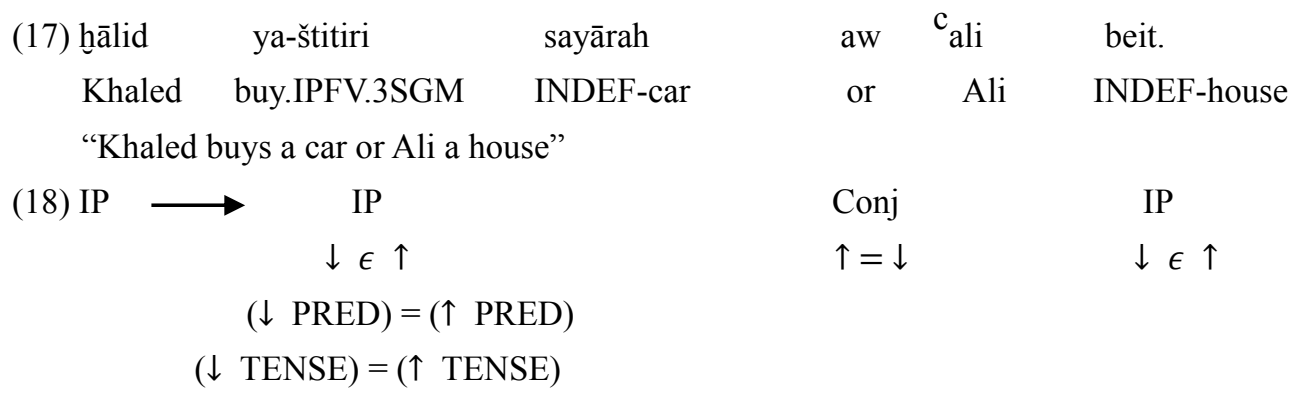

Example (17) illustrates gapping in this dialect, where the verb in the second conjunct is deleted. The phrase structure rule in (18) spreads the PRED and TENSE of the verb in the first conjunct over the coordinate structure. In this analysis, both conjuncts are complete. The rule in (18) licenses the c-structure in (19.a), which can be related to the f-structure in (19.b) below. 

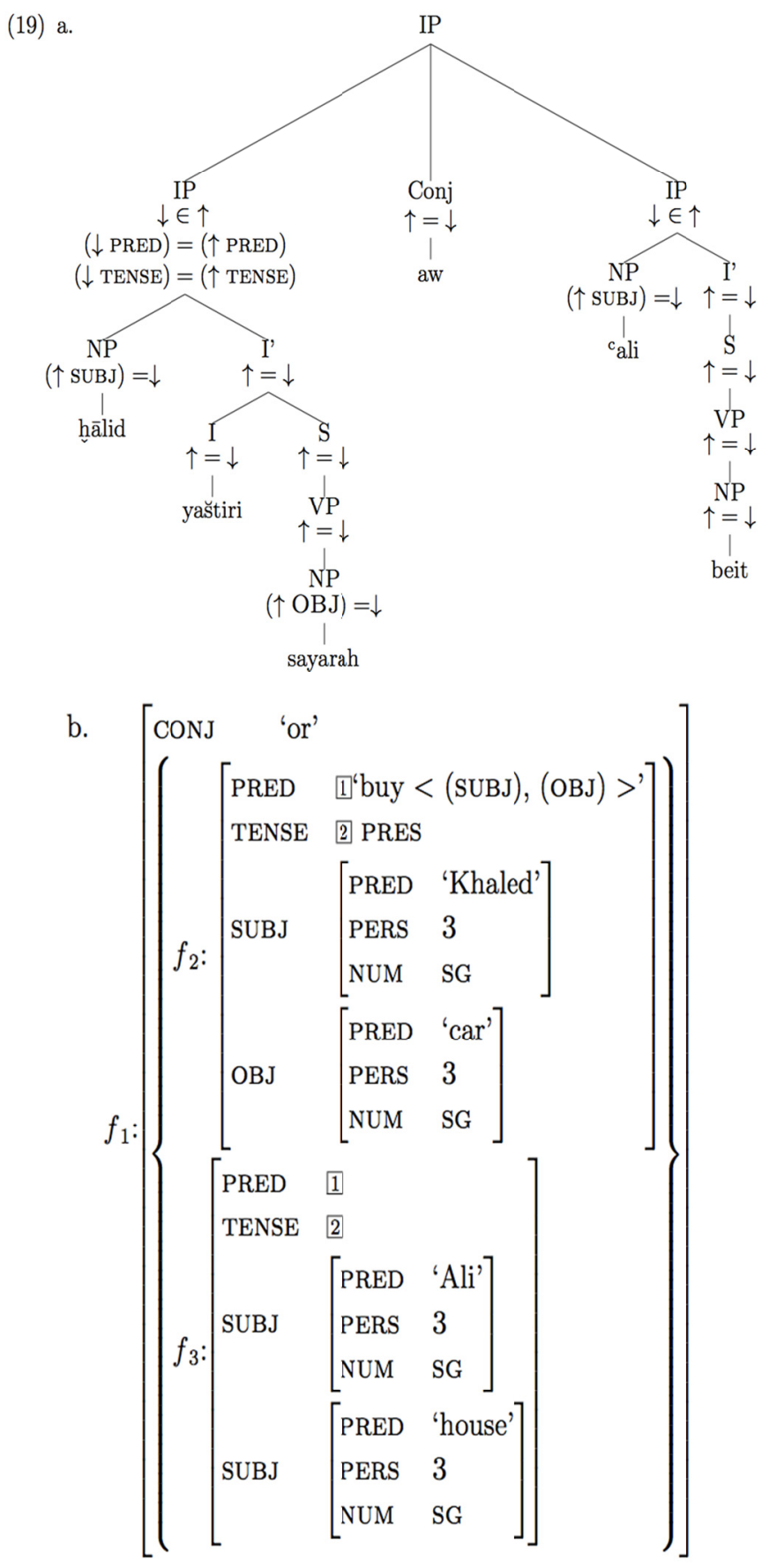

Alzaidi (2010) states that this approach is able to analyze gapping in the Hijazi dialect. The PRED and tense of the verb in the first conjunct are distributed to the following conjunct in example (17). Therefore, the f-structure shown in (12.b) above is well-formed, meaning that it is complete (Note 2), coherent (Note 3) and consistent (Note 4).

However, the present paper argues against this analysis, suggesting that adopting such analysis for gapping constructions is problematic. The problem is that the verb in the first conjunct has been used twice: the verb requires a subject and an object; both are available in the first conjunct; but the same verb governs other arguments in the second conjunct. Therefore, this paper claims that the f-structure is not coherent in this analysis and therefore is not well-formed. The f-structure is not coherent because the functions in the second conjunct in this analysis are not required by a predicate. In the next section, we will propose a different analysis that is able to account for gapping better than previous analyses in LFG. 


\section{Suggested Analysis}

In this paper, we believe that neither analysis in LFG discussed above is the best one for gapping. As mentioned above, the split analysis adopted by Maxwell \& Manning (1996) is unable to account for all examples of gapping in MSA and other languages. Their analysis can account for an example such as (7) above, repeated in (20) below, but it cannot account for an example such as (12), repeated in (21), where only the predicate is shared between the two conjuncts.

(20) John introduced David to Mary and Louisa to Chris.

(21) John likes Mary and David Sue.

We have noted another problem that split analysis shares with function spreading. In particular, we posit that both analyses do not align well with one of the main principles in LFG, which is the requirement of a predicate. In this paper, we claim that the f-structures proposed by both analyses are not well-formed because they are not coherent. In LFG, the f-structure must be coherent, and it is coherent if all the arguments in this f-structure are required by a predicate. Split analysis assumes that the first predicate is used twice in both conjuncts, and this assumption yields a predicate that requires two arguments with four arguments. Similarly, function spreading assumes that the predicate is spread over the coordinate structure and is used in this way twice in the first conjunct and in the second conjunct. The predicate requires two arguments in the shown examples above, but it is used with four arguments.

To solve these problems in both analyses, this paper claims that another omitted predicate, which is the same as the overt predicate in the first conjunct, should be available in the analysis of this phenomenon. Thus, we develop an analysis in LFG that assumes that there is an omitted predicate in the second conjunct in gapping constructions. This analysis is supported by the behavior of coordination in general, which is used to test for constituency, meaning that a conjunction in a coordinate structure should coordinate the same constituents. Thus, we should assume that the second conjunct in gapping contains the same elements available in the first conjunct and that the verb is the most important element.

To propose an analysis in LFG that allows for an omitted predicate in the second conjunct, we suggest that this analysis use the so-called empty category, whereby the omitted verb is analyzed as a null verb that indicates a tense and requires arguments. Simpson (1991) uses the empty category to analyze the null auxiliary in Warlpiri. In the phrase structure rules, the empty category is represented by the symbol $\in$.

The example in (2.a), repeated in (22.a) below, illustrates gapping in MSA. Notably, the verb in the second conjunct can be omitted, as shown in (22.a), and it can also appear, as shown in (3.a), repeated in (22.b) below:

$\begin{array}{llllll}\text { (22) a. qābala } & \text { fāris-un hāmid-an } & \text { wa zayd-un } & \text { Payma-an. } \\ \text { Meet.PFV.3SGM } & \text { Faris-NOM Hamed-ACC } & \text { and } & \text { Zayd-NOM } & \text { Ayman-ACC } \\ \text { "Faris met Hamed and Zayd Ayman" } & & & & \\ \text { b. qābala } & \text { făris-un hāanid-an } & \text { wa } & \text { qābala } & \text { zayd-un } & \text { Payma-an. } \\ \text { Meet.PFV.3SGM } & \text { Faris-NOM Hamed-ACC } & \text { and } & \text { Meet.PFV.3SGM } & \text { Zayd-NOM } & \text { Ayman-ACC } \\ \text { "Faris met Hamed and Zayd met Ayman" } & & & & \end{array}$

The suggested rule should cover the two possibilities, where the verb in the second conjunct does not appear, as shown in (22.a), and where it does appear, as shown in (22.b). We need to create a rule for I', where verbs should appear in MSA. This rule should indicate that the predicate can appear under I' or can be omitted. Such a rule is possible in LFG when a disjunction and the symbol $\in$, which represents the empty category, are used. The following rule fulfills these requirements successfully. This rule contains a disjunction, showing that two possible options are available for I' in this analysis. In the first option, the verb may appear and will be represented in the c-structure without any problems, and this option is appropriate for example (22.b). In the second option, the verb is omitted and represented as an empty category that indicates the tense and its requirements as a usual predicate; however, it will not appear in the c-structure in this case, which is appropriate for example (22.a) above. This rule should represent the I' in the second conjunct.

(23) I'

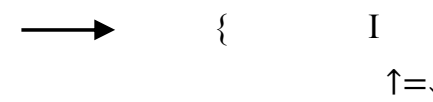

$$
\begin{array}{lcc}
\text { I } & \in \\
\uparrow=\downarrow & (\uparrow \text { TENSE })=(\downarrow \text { TENSE }) \\
& & (\uparrow \text { PRED })=(\downarrow \text { PRED })
\end{array}
$$


As stated above, when the predicate is omitted in an example such as (22.a), it will not be represented in the c-structure but will appear in the f-structure. Thus, the requirements of this predicate, which are two arguments, serve the two functions of the subject and the object, and they are available in the second conjunct. The question then is how we represent this empty category in the lexical entry. We suggest the following lexical entry for the omitted verb, where the omitted verb is represented by the sample $\in$, as shown below

$(24) \in \mathrm{I} \prime \quad(\uparrow \mathrm{PRED})=$ "meet $<(\mathrm{SUBJ}),(\mathrm{OBJ})>$ "

$$
(\uparrow \text { TENSE })=\text { PAST }
$$

The suggested f-structure for an example such as (22.a) above is shown in (25) below:

\begin{tabular}{|c|c|}
\hline CONJ & 'and' \\
\hline $\int[\mathrm{PRED}$ & 'meet $<($ SUBJ $),($ OBJ $)>']$ \\
\hline TENSE & PAST \\
\hline SUBJ & {$[$ PRED 'Faris' } \\
\hline OBJ & {$[$ PRED 'Hamed'] } \\
\hline PRED & 'meet $<($ SUBJ $),($ OBJ $)>']$ \\
\hline TENSE & PAST \\
\hline SUBJ & {$[$ PRED 'Zayd'] } \\
\hline OBJ & {$[$ PRED 'Ayman'] } \\
\hline
\end{tabular}

This f-structure has two predicates: the first predicate is the overt verb qābala ("met") in the first conjunct, and the second predicate is the null verb qäbala ("met") in the second conjunct. Both predicates appear in the f-structure, and the tense of both predicates (the past tense) is represented in the embedded f-structures of both conjuncts.

This analysis can account for this phenomenon in MSA and other languages as well. The main advantage of this analysis is to create a well-formed f-structure that follows the coherency condition and avoids the problems in previous analyses that use a predicate twice by spreading it from one conjunct to another. Additionally, the presence of the predicate in the second conjunct in example (22.b) means that the predicate is omitted in example (22.a), thus motivating the analysis suggested in this paper.

\section{Conclusion}

This paper has discussed gapping in MSA within the LFG framework. This paper focuses on an analysis in LFG that can account for this phenomenon in MSA and other languages. This study argues against previous analyses in LFG, where gapping is analyzed by using a split category in Maxwell and Manning (1996) or by assuming that the verb in the first conjunct should be spread over the entire coordinate structure via function spreading. This paper suggests that both analyses have a serious problem in allowing the verb in the initial conjunct to be used twice in the first conjunct and then in the second conjunct. Instead, our analysis assumes that the verb in the second conjunct should be analyzed as a null verb that has a requirement and tense feature in its local clause.

\section{References}

Alzaidi, M. S. (2010). Gapping and right node raising: An LFG approach. Ph. D. thesis, MA Thesis. University of Essex.

Bresnan, J. (2001). Lexical-Functional Syntax. Oxford: Blackwell Publishers.

Bresnan, J., Kaplan, R. M., \& Peterson, P. G. (1985). Coordination and the flow of information through phrase structure. MS, Xerox PARC.

Dalrymple, M. (2001). Lexical functional grammar, 42. New York: Academic Press. https://doi.org/10.1163/9781849500104

Frank, A. (2002). A (discourse) functional analysis of asymmetric coordination. In M. Butt \& T. H. King (Eds.), Proceedings of the LFG02 Conference. Stanford, CA. CSLI Publications. Retrieved from 
http://www-csli.stanford.edu/publications.

Gleitman, L. R. (1965). Coordinating conjunctions in English. Language, 41(2), 260-293. https://doi.org/10.2307/411878

Kaplan, R. M., \& J. Bresnan (1982). Lexical Functional Grammar: a Formal System for Grammatical Representation. In J. Bresnan (Ed.), The Mental Representation of Grammatical Relations (pp. 173-282). Cambridge, MA: MIT Press.

Kaplan, R. M., \& Maxwell III, J. T. (1988). Constituent coordination in Lexical-Functional Gram- mar. In Proceedings of the 12th International Conference on Computational Linguistics (COL-ING 88), 1, pp. 303-305, 199-210.

Lakoff, G., \& Ross, J. R. (1970). Gapping and the order of constituents. Progress in linguistics: A collection of papers, 43, 249.

Maxwell, J. T., \& Manning, C. D. (1996). A theory of non-constituent coordination based on finite-state rules. In Proceedings of $L F G, 96$.

Rouveret, A. (1994). Syntaxe du gallois. Paris, France: Editions CNRS.

Sadler, L. (2006). Function spreading in coordinate structures. Lingua 116(11), 1777-1806. https://doi.org/10.1016/j.lingua.2004.09.005

Simpson, J. (1991). Warlpiri Morpho-Syntax: A Lexicalist Approach. Dordrecht: Kluwer Academic Publishers. https://doi.org/10.1007/978-94-011-3204-6

\section{Notes}

Note 1. There is no need for the c-structure and f-structure because the main point in this analysis can be shown through the rule.

Note 2. The f-structure is complete if it contains all the grammatical functions required by the predicate in this f-structure (see Kaplan \& Bresnan, 1982).

Note 3. The f-structure is complete if it contains all the grammatical functions required by the predicate in this f-structure (see Kaplan \& Bresnan, 1982).

Note 4. The f-structure is consistent if almost any attribute has one value (see Kaplan \& Bresnan, 1982).

\section{Copyrights}

Copyright for this article is retained by the author(s), with first publication rights granted to the journal.

This is an open-access article distributed under the terms and conditions of the Creative Commons Attribution license (http://creativecommons.org/licenses/by/4.0/). 